\title{
Optical Readout of the Néel Vector in the Metallic Antiferromagnet $\mathrm{Mn}_{2} \mathrm{Au}$
}

\author{
Vladimir Grigorev, ${ }^{1,2}$ Mariia Filianina, ${ }^{1,2}$ Stanislav Yu. Bodnar, ${ }^{1,3}$ Sergei Sobolev, ${ }^{1}$ \\ Nilabha Bhattacharjee $\odot,{ }^{1}$ Satya Bommanaboyena, ${ }^{1}$ Yaryna Lytvynenko $\odot,{ }^{1}$ Yurii Skourski, ${ }^{4}$ \\ Dirk Fuchs, ${ }^{5}$ Mathias Kläui, ${ }^{1,2}$ Martin Jourdan, ${ }^{1}$ and Jure Demsar ${ }^{1,2, *}$ \\ ${ }^{1}$ Institute of Physics, Johannes Gutenberg University, 55128 Mainz, Germany \\ ${ }^{2}$ Graduate School of Excellence Materials Science in Mainz, 55128 Mainz, Germany \\ ${ }^{3}$ Walter Schottky Institut and Physics Department, Technische Universität München, 85748 Garching, Germany \\ ${ }^{4}$ Dresden High Magnetic Field Laboratory (HLD-EMFL), Helmholtz-Zentrum Dresden-Rossendorf, 01328 \\ Dresden, Germany \\ ${ }^{5}$ Institute for Quantum Materials and Technologies, Karlsruhe Institute of Technology, 76344 \\ Eggenstein-Leopoldshafen, Germany
}

(Received 8 April 2021; accepted 23 June 2021; published 15 July 2021)

\begin{abstract}
Metallic antiferromagnets with broken inversion symmetry on the two sublattices, strong spin-orbit coupling, and high Néel temperatures offer alternative opportunities for applications in spintronics. Especially $\mathrm{Mn}_{2} \mathrm{Au}$, with a high Néel temperature and high conductivity, is particularly interesting for real-world applications. Here, manipulation of the orientation of the staggered magnetization, (i.e., the Néel vector) by current pulses was recently demonstrated, with the readout limited to studies of anisotropic magnetoresistance or X-ray magnetic linear dichroism. Here we report on the in-plane reflectivity anisotropy of $\mathrm{Mn}_{2} \mathrm{Au}(001)$ films, which are Néel vector aligned in pulsed magnetic fields. In the near-infrared region, the anisotropy is approximately $0.6 \%$, with higher reflectivity for the light polarized along the Néel vector. The observed magnetic linear dichroism is about 4 times larger than the anisotropic magnetoresistance. This suggests the dichroism in $\mathrm{Mn}_{2} \mathrm{Au}$ is a result of the strong spin-orbit interactions giving rise to anisotropy of interband optical transitions, which is in line with recent studies of electronic band structure. The considerable magnetic linear dichroism in the near-infrared region could be used for ultrafast optical readout of the Néel vector in $\mathrm{Mn}_{2} \mathrm{Au}$.
\end{abstract}

DOI: 10.1103/PhysRevApplied.16.014037

\section{INTRODUCTION}

There is increasing interest in antiferromagnetic (AFM) materials as active elements in future spintronics devices, including data-storage applications [1-5]. This is motivated by the absence of net magnetization and the related stray fields, which limit the minimum distance between two bits, and thus leads to higher storage density compared with ferromagnets. Moreover, the inherently fast spin dynamics in antiferromagnets, which occurs in the terahertz range [6-10], is orders of magnitude faster then typical spin dynamics in ferromagnetic materials (gigahertz range) [11]. The absence of net magnetization, however, makes the manipulation and readout of magnetic order, which is given by the staggered magnetization (i.e., the Néel vector) generally difficult.

Recently, two fully compensated metallic antiferromagnets, CuMnAs and $\mathrm{Mn}_{2} \mathrm{Au}$, have been the focus of

\footnotetext{
*demsar@uni-mainz.de
}

research. Here, the specific crystal and magnetic structure in combination with large spin-orbit coupling enable current-driven manipulation of the Néel vector [12]. In particular, it was suggested that application of electric current pulses on the order of $10^{8}-10^{9} \mathrm{~A} / \mathrm{cm}^{2}$ can rotate the Néel vector due to the bulk Néel spin-orbit torques [12]. Such current-driven switching was indeed demonstrated in the metallic antiferromagnets CuMnAs [13-15] and $\mathrm{Mn}_{2} \mathrm{Au}$ [16-18], with pulse durations down to picoseconds, achieved by driving currents with pulsed terahertz radiation [19]. The high Néel temperature (approximately $1500 \mathrm{~K})$ and high electrical conductivity $[20,21]$ make $\mathrm{Mn}_{2} \mathrm{Au}$ particularly interesting for real-world applications. In $\mathrm{Mn}_{2} \mathrm{Au}$ the current pulses were shown to reorient only a fraction (up to 30\%) of the domains [17], implying further studies of the switching mechanisms as well as alternative approaches are required.

The absence of net magnetization makes the efficient readout of the direction of the Néel vector challenging. Indeed, electrical readout via the anisotropic magnetoresistance (AMR) and the planar Hall effect is well established 
$[13,16]$. However, recent studies showed that in $\mathrm{Mn}_{2} \mathrm{Au}$ the AMR does not exceed $0.15 \%$ [22]. Moreover, the readout time in the AMR detection scheme is inherently limited by the electronics to timescales orders of magnitude longer than the intrinsic switching timescales [19]. Thus, using optical methods, which would enable fast readout on the femtosecond scale, should be explored [23,24]. While linear magneto-optic effects are commonly used to investigate magnetization and its dynamics in ferromagnets and ferrimagnets [25,26], as well as in noncollinear antiferromagnets [27], the presence of quadratic magneto-optical effects (Cotton-Mouton/Voigt effect) was demonstrated in several insulating antiferromagnets [7,28-30]. To probe the small changes in the refractive index due to the quadratic magneto-optical effects, polarimetry studies are commonly performed in the transmission geometry, since the changes in the optical phase accumulate over the optical path length within the material [28-30]. For metallic collinear AFM materials, with optical penetration depths on the order of tens of nanometers, the corresponding changes are small. Thus, for CuMnAs another approach was recently demonstrated [31,32]. Here, the thermomodulation aspect of the optical pump-probe technique was used to detect small photoinduced changes in rotation of the polarization of the optical probe beam. Since such changes depend on the polarization of light with respect to the Néel vector, probe-polarization dependence is used to determine the direction of the Néel vector [31,32]. Indeed, a similar approach was recently used to study dynamics in metallic $\mathrm{AFM} \mathrm{Fe} \mathrm{F}_{2} \mathrm{As}$ [33] and insulating $\mathrm{CoO}$ [34]. Finally, several recent reports demonstrated large magneto-optical contrast also in ultrathin $\mathrm{NiO}$ [35-37] and $\mathrm{CoO}$ [38] films; however, in this case the origin of the magneto-optical contrast is in large magnetostriction.

In $\mathrm{Mn}_{2} \mathrm{Au}$, a recent angular-resolved-photoemission study of Néel vector-aligned $\mathrm{Mn}_{2} \mathrm{Au}$ films demonstrated the breaking of the $C_{4 z}$ symmetry, a consequence of antiferromagnetic order and strong spin-orbit interaction [39]. A pronounced in-plane anisotropy of the electronic band structure was observed up to a binding energy of a few electronvolts [39]. The existence of flat bands at a binding energy of $1.5 \mathrm{eV}$ and their calculated anisotropy may provide means to detect a substantial magnetic linear dichroism (MLD) in the near-infrared (NIR) region.

Here we report on near-infrared MLD measurements on $\mathrm{Mn}_{2} \mathrm{Au}$ thin films performed in reflectance geometry at room temperature. To determine the magnitude of the MLD, we investigate $\mathrm{Mn}_{2} \mathrm{Au}$ films whose staggered magnetization (Néel vector) is aligned with use of a pulsed magnetic field [22]. The MLD in the NIR region is found to be approximately $0.6 \%$, about 4 times larger than the observed anisotropic magnetoresistance in $\mathrm{Mn}_{2} \mathrm{Au}$ [22]. The comparatively large MLD suggests it originates from the anisotropy in the interband absorption as a result of $C_{4 z}$-symmetry breaking.

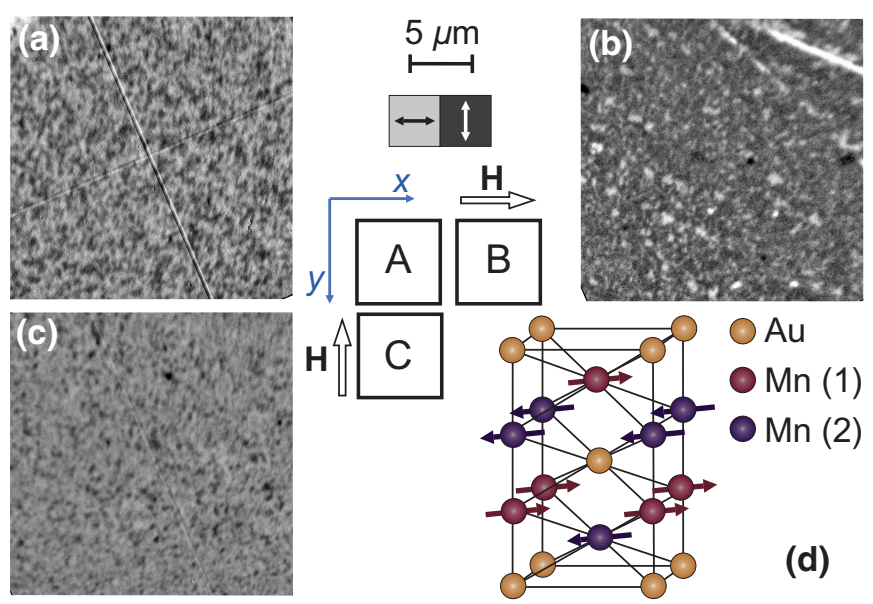

FIG. 1. The sample layout and the XMLD PEEM images of samples A-C, shown in (a)-(c), respectively. $\mathrm{Mn}_{2} \mathrm{Au}$ grows epitaxially with the [110] and [110] axes parallel to the substrate edges, which are along the $[010]_{s}(x)$ and $[211]_{s}(y)$ axes of the r-cut sapphire. Sample A is as grown, while samples B and $\mathrm{C}$ are orthogonally polarized in the $60-\mathrm{T}$ pulsed magnetic field. The directions of the applied magnetic field and the corresponding Néel-vector directions are shown in the center, together with the scale of the XMLD PEEM images. The contrast shows the two types of magnetic domains with the Néel vector along the two orthogonal easy axes: [110] and [1 $\overline{1} 0]$. In the as-prepared sample (A), roughly equal volume fractions of both types of domains are observed, while the aligned samples (B and C) display mostly one type of domain. (d) The unit cell of $\mathrm{Mn}_{2} \mathrm{Au}$, with spin orientations in adjacent layers shown by arrows.

\section{RESULTS AND DISCUSSION}

\section{A. $M n_{2} A u$ thin films}

$\mathrm{Mn}_{2} \mathrm{Au}$ has a body-centered tetragonal crystal structure, whose unit cell is depicted in Fig. 1(d). It is an easy-plane (001) antiferromagnet with a strong out-of plane hard axis and weak in-plane magnetic anisotropy. The spin orientations in adjacent layers are represented by arrows in Fig. 1(d), with the Néel vector pointing along the easy [110] directions $[20,21,40]$.

The $c$-axis epitaxial $\mathrm{Mn}_{2} \mathrm{Au}$ thin film is grown on an r-cut $\mathrm{Al}_{2} \mathrm{O}_{3}(1102)$ substrate, with a lateral size of $10 \times$ $10 \mathrm{~mm}^{2}$ and a thickness of $530 \mu \mathrm{m}$ by radio-frequency magnetron sputtering at $600^{\circ} \mathrm{C}$ (see Refs. [21,37] for fabrication details). To ensure epitaxial growth, a 45 -nm-thick $\mathrm{Mn}_{2} \mathrm{Au}$ film is deposited on a 20 -nm-thick $\mathrm{Ta}(001)$ buffer layer. To protect the surface, a 2-nm Al layer is deposited on $\mathrm{Mn}_{2} \mathrm{Au}$, forming an aluminum oxide capping layer. $\mathrm{Mn}_{2} \mathrm{Au}$ grows epitaxially with the [110] and [110] axes parallel to the substrate edges, which are along the $[010]_{S}$ and $[211]_{s}$ directions of $\mathrm{r}$-cut $\mathrm{Al}_{2} \mathrm{O}_{3}$. As elaborated in Appendix $\mathrm{B}$, the $c$ axis of $\mathrm{Mn}_{2} \mathrm{Au}$ films grown on $\mathrm{r}$-cut $\mathrm{Al}_{2} \mathrm{O}_{3}$ is tilted by $2-3^{\circ}$ toward the $[010]_{s}$ direction. 
As previously demonstrated $[22,39,41,42]$, the application of a magnetic field exceeding $30-50 \mathrm{~T}$ results in the alignment of the Néel vector along one of the easy axes, perpendicular to the applied magnetic field [41]. While such a "polarization" of the film may be incomplete (i.e., there is still a small fraction of orthogonally polarized domains), it is quasipermanent $[22,39,41]$.

To perform systematic measurements, the as-grown sample is cut into $5 \times 5 \mathrm{~mm}^{2}$ pieces. The three pieces investigated are labeled A-C. Sample A is in the asprepared state (i.e., in a multidomain configuration) and is used as a reference. Samples B and C are Néel vector aligned $[22,39,41]$ in a pulsed magnetic field of 60 $\mathrm{T}$ along the different yet crystallographically equivalent $\{110\}$ directions, as shown in the middle panel in Fig. 1. Throughout this work the default film orientation is such that $x \|[010]_{s}$ and $y \|[211]_{s}$.

\section{B. X-ray magnetic linear dichroism in polarized $\mathrm{Mn}_{2} \mathrm{Au}$ films}

Before optical studies the AFM domain patterns of all samples are first imaged by x-ray magnetic linear dichroism photoemission electron microscopy (XMLD PEEM). The XMLD PEEM imaging of the AFM domains is performed at room temperature at the SIM beamline of the Swiss Light Source. The sample is illuminated by linearly polarized $\mathrm{x}$ rays with the polarization in the sample plane and an angle of incidence of $16^{\circ}$. The XMLD contrast is obtained by use of the two-energy mode, as described in detail in Ref. [42]. The energies $E_{1}=639 \mathrm{eV}$ and $E_{2}=$ $638.2 \mathrm{eV}$ are used, which correspond to the maximum and minimum of the XMLD [42].

The XMLD PEEM images of all samples are shown in Fig. 1, demonstrating that samples $\mathrm{B}$ and $\mathrm{C}$ have an orthogonally oriented Néel vector. In particular, each of the polarized samples has mostly one type of AFM domain, with narrow wormlike domains of the other type, similarly to earlier reports $[41,42]$. Sample A, on the other hand, has approximately equal densities of the domains of both types. Hence, sample A is used as a reference in the optical dichroism measurements.

\section{Magnetic linear dichroism in the near-infrared region}

\section{General symmetry considerations}

In a tetragonal easy-plane system such as $\mathrm{Mn}_{2} \mathrm{Au}$, a symmetry-based phenomenological model of the quadratic magneto-optical effect generally implies two possible contributions to the MLD: an isotropic and an anisotropic contribution [28]. The isotropic contribution does not depend on the direction of the Néel vector within the plane, and can be observed when light is propagating along the twofold symmetry axis of the crystal (here we are considering the crystal symmetry only). The anisotropic MLD does depend on the direction of the Néel vector [29] and is present when the light propagates along the fourfold symmetry axis. It is the anisotropic component that can be used to determine the Néel-vector orientation in a collinear antiferromagnet.

The corresponding dielectric tensor of an antiferromagnet with a tetragonal crystal structure and the Néel vector perpendicular to the fourfold symmetry axis (i.e., within the easy $a-b$ plane) can be written as [28]

$$
\begin{aligned}
& \epsilon_{x x}=\epsilon_{\perp}+\lambda_{1} \vec{L}^{2}+\frac{1}{2} \lambda_{3}\left(L_{x}^{2}-L_{y}^{2}\right)-2 \lambda_{4} L_{x} L_{y}, \\
& \epsilon_{y y}=\epsilon_{\perp}+\lambda_{1} \vec{L}^{2}-\frac{1}{2} \lambda_{3}\left(L_{x}^{2}-L_{y}^{2}\right)+2 \lambda_{4} L_{x} L_{y}, \\
& \epsilon_{z z}=\epsilon_{\|}+\lambda_{2} \vec{L}^{2}, \\
& \epsilon_{x y}=\epsilon_{y x}=\frac{1}{2} \lambda_{3}\left(L_{x}^{2}-L_{y}^{2}\right)+2 \lambda_{4} L_{x} L_{y}, \\
& \epsilon_{x z}=\epsilon_{z x}=\epsilon_{y z}=\epsilon_{z y}=0,
\end{aligned}
$$

where $\epsilon_{\perp}$ and $\epsilon_{\|}$are the in-plane and out-of-plane dielectric constants in the absence of magnetic order, $\vec{L}$ is the Néel vector, and $\lambda_{i}$ are the phenomenological coefficients related to the MLD. Given the two possible directions of the Néel vector in $\mathrm{Mn}_{2} \mathrm{Au}$, we choose the $z$ axis to be along the fourfold [001] axis, while $x$ and $y$ coincide with the [110] and [1 $\overline{1} 0]$ crystallographic axes. The difference between $\epsilon_{x x}$ or $\epsilon_{y y}$ and $\epsilon_{z z}$ is governed by $\epsilon_{\perp}-\epsilon_{\|}$, yet also includes the component proportional to $\vec{L}^{2}$, i.e., the so-called isotropic component of the MLD (the term proportional to $\vec{L}^{2}$ is likely negligible compared with $\epsilon_{\perp}-$ $\left.\epsilon_{\|}\right)$. The difference $\epsilon_{\perp}-\epsilon_{\|}$may lead to the reflectivity anisotropy in the non-normal incidence configuration (see Appendix B).

\section{Experimental approach}

The goal of the experiment is to determine the anisotropic magnetic contribution to dichroism, given by $\epsilon_{x x}-\epsilon_{y y}$, which is proportional to the phenomenological constants $\lambda_{3}$ and $\lambda_{4}$ and depends on the (in-plane) Néelvector orientation. Such a MLD is expected to be in the range of $10^{-3}$ and could be overshadowed by extrinsic nonmagnetic effects such as strain. In particular, in the case of an additional lattice distortion in the $a-b$ plane due to strain, $\epsilon_{\perp}$ (and $\lambda_{1}$ as well) would be different for the $x$ and $y$ directions, leading to reflectivity anisotropy in the $a-b$ plane unrelated to the magnetic order (see the discussion in Appendix B). Furthermore, such extrinsic contributions may vary from sample to sample due to a slight variation of substrate properties such as miscut. Thus, to unambiguously determine the MLD, measurements on different magnetically aligned parts of the same film are performed.

Optical dichroism is studied with the setup shown in Fig. 2(a); for details see Appendix A. The measurements are performed at room temperature. We use a 852-nm laser 

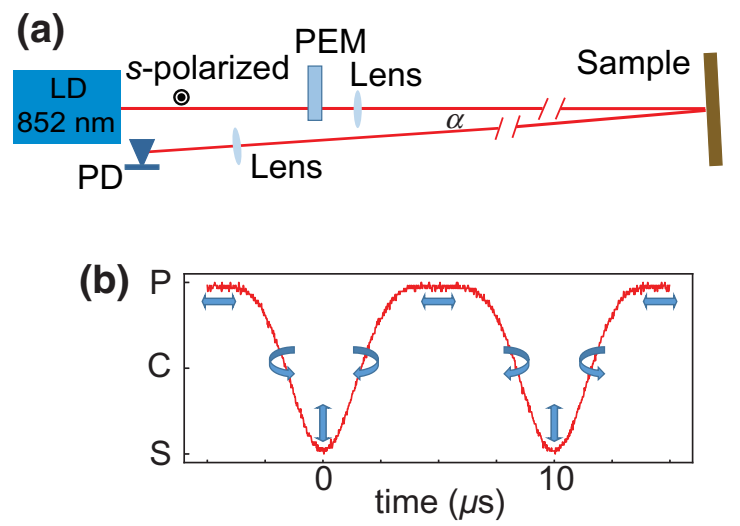

FIG. 2. (a) The experimental setup (top view). The laser diode (LD) is vertically polarized ( $s$-polarized). The polarization is modulated by the PEM operating at $50 \mathrm{kHz}$. The distance between the LD and the sample is about $2.5 \mathrm{~m}$, while the photodiode (PD) is mounted about $3 \mathrm{~cm}$ from the laser output, giving an incidence angle of $\alpha / 2 \approx 0.34^{\circ}$. (b) Time evolution of the light intensity on the PD when a polarizer is placed in front of the PD selecting the horizontal ( $p$-polarized) signal (the modulation period is $10 \mu \mathrm{s})$. The blue arrows represent the polarization states at given times, varying between vertical $(s)$ and horizontal $(p)$ polarizations, which are along the [110] and [1 $\overline{10}]$ crystalline axes of $\mathrm{Mn}_{2} \mathrm{Au}$. C, circularly polarized light; $\mathrm{P}, p$-polarized light; $\mathrm{S}, s$-polarized light.

diode and a photoelastic modulator (PEM) to modulate the polarization of incoming light between the two orthogonal linear polarizations: $s$-polarized (vertical) and $p$-polarized (horizontal). The $\mathrm{Mn}_{2} \mathrm{Au}$ films are mounted such that their easy axes are along the horizontal and vertical directions, which correspond to the $x$ and $y$ directions of $\epsilon_{i j}$. The light reflected from the sample is focused onto a photodiode and the ac signal is recorded with a digital oscilloscope. Measurements at multiple positions on each of the three samples are performed (the laser-spot diameter on the sample is about $500 \mu \mathrm{m}$ ) and averaged to minimize systematic errors.

\section{Disentanglement of the MLD and structural dichroism}

In our experimental geometry, with $x$ corresponding to horizontal light polarization and $y$ corresponding to vertical light polarization, the MLD, given by $\epsilon_{x x}-\epsilon_{y y}$ in Eq. (1), should result in a difference between the reflectivities of the $s$-polarized and $p$-polarized light. However, weak modulation of the intensity of the reflected light is also expected due to effects related to the operation of the PEM, as well as structural/strain anisotropy. Thus, the modulation of light intensity on the photodiode, $\Delta I=\Delta R / R$ (the reflectivity, $R$, of $\mathrm{Mn}_{2} \mathrm{Au}$ at $852 \mathrm{~nm}$ is $0.52 \pm 0.01$ ), can be expressed as

$$
\Delta I_{i}(\phi)=D_{0} P(\phi)+D_{\mathrm{mag}}^{i} P(\phi)+D_{\mathrm{str}}^{i} P(\phi),
$$

where $D_{0}$ is the contribution to the signal related to the PEM operation and slight misalignment of the optical components (present also when we measure an uncoated gold mirror as a reference), $D_{\text {mag }}^{i}$ is the amplitude related to the MLD of the sample, where $i=A-C$, and $D_{\text {str }}^{i}$ is the amplitude of the dichroism caused by structural effects, such as strain. We assume that in the first approximation the structural/strain dichroism is decoupled from $D_{\mathrm{mag}}^{i}$ (i.e., the strain does not affect the Néel vector). Thus, we assume $D_{\mathrm{str}}^{i}$ is independent of the Néel-vector direction but depends on the sample orientation (see also Appendix B). Finally, $P(\phi)$ is a periodic function describing the polarization state of light after passing through the PEM; see Fig. 2(a). Since the same experimental geometry is used for all samples, $D_{0}$ is the same in all measurements. Moreover, given samples A-C are cut from the same film, $D_{\text {str }}^{i}$ should be identical for all samples when they are mounted along the same direction with respect to the asgrown sample. Conversely, $D_{\mathrm{str}}^{i}$ should change sign when the sample is rotated by $90^{\circ}$ around the $C_{4}$ axis, i.e., $D_{\mathrm{str}}^{i}=-D_{\mathrm{str}}^{i(90)}$. When we consider the MLD, $D_{\mathrm{mag}}^{A} \approx 0$, given the multidomain state on the length scale of the laserspot size of $500 \mu \mathrm{m}$. Finally, if we take into account the orthogonal direction of the Néel vector in the two magnetically aligned samples, it follows that $D_{\mathrm{mag}}^{B}=-D_{\mathrm{mag}}^{C}$. Under these assumptions the structural and magnetic contributions can be disentangled by the following referenced measurements:

$$
\begin{gathered}
D_{\mathrm{mag}} P(\phi) / 2=\Delta I_{B}(\phi)-\Delta I_{A}(\phi)=\Delta I_{A}(\phi)-\Delta I_{C}(\phi), \\
D_{\mathrm{str}} P(\phi)=\Delta I_{A}(\phi)-\Delta I_{A}^{(90)}(\phi)=\Delta I_{B}(\phi)-\Delta I_{C}^{(90)}(\phi) .
\end{gathered}
$$

\section{Near-infrared magnetic linear dichroism in $\mathrm{Mn}_{2} \mathrm{Au}$}

Figure 3 presents the results of dichroism measurements performed in sequence under identical conditions. The dashed black line, representing the evolution of the polarization state - see Fig. 2(b) - is added to all panels as a guide for the eye. Figure 3(a) presents the raw data, $\Delta I_{i}(\phi)$, taken on samples A-C. The complicated shape of the raw signal is largely a result of the optical elements of the setup, mainly the PEM. The PEM acts as a $\lambda \times \delta$ wave plate, where $\delta$ is modulated between $-1 / 2$ and $1 / 2$. This results in a sequence of polarization states $s-p-s-p-s$ within one modulation period of the PEM (20 $\mu \mathrm{s})$. Between $s$ and $p$ polarizations, the light is circularly/elliptically polarized. Because of imperfections and asymmetry between the optical properties of the squeezed and elongated PEM crystal, a modulation of the signal at $50 \mathrm{kHz}$ is also observed.

To obtain the MLD component we first subtract the signal of the reference sample (as prepared, sample A) from signals obtained on the magnetically polarized samples B and C, following Eq. (3). Moreover, as the MLD should 

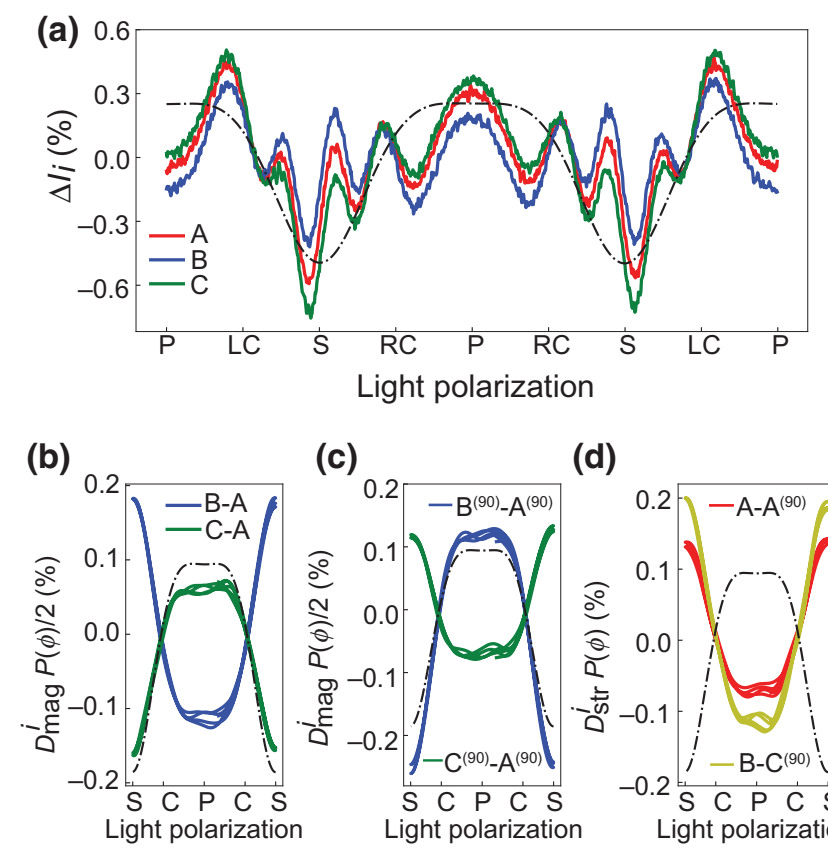

(d)

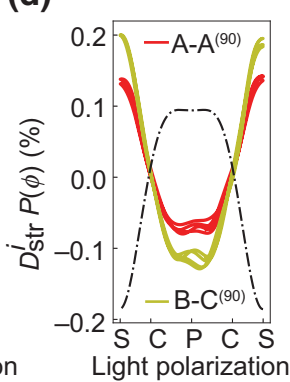

FIG. 3. Results of reflectivity anisotropy studies of $\mathrm{Mn}_{2} \mathrm{Au}$ films with different orientations of the magnetic order. (a) The raw reflectivity modulation signals from all three samples, oriented in the same growth direction (raw data obtained on samples rotated by $90^{\circ}$ are not shown). The time window here is $20 \mu \mathrm{s}$. (b)-(d) The differential signals disentangling the MLD and structural contributions to reflectivity variation according to Eqs. (3) and (4). Here the traces are folded into the $10-\mu$ s time window to emphasize the lack of circular dichroism, which should have a $50-\mathrm{kHz}$ component. The modulation in (b),(c) is a result of the MLD, while the modulation in (d) reflects the structural component of dichroism. The modulation of reflectivity is recorded with a digital oscilloscope in the ac coupling mode; thus, the zero baseline is automatically determined by the oscilloscope as the mean value of the periodic signal. Because of the asymmetry of the signal, the zero level does not correspond to the arithmetic average of the maximum and minimum. The asymmetry is a result of PEM operation, where the flat regions [here the light is $p$-polarized; see Fig. 2(b)] correspond to the maximal retardation of the photoelastic modulator. LC, left-circularlypolarized light; P, p-polarized light; S, s-polarized light; RC, right-circularly-polarized light.

give rise to a periodic modulation of reflectivity at the second harmonic of the PEM frequency, we fold the data recorded over two periods into the $10-\mu$ s time window. The resulting variations are shown in Fig. 3(b). The differential signals do not have the complicated shape of the raw data as in Fig. 3(a), demonstrating that subtraction efficiently cancels out all system-related modulations. Indeed, the shape of the differential signal follows the shape of the reference curve (dashed line). Furthermore, the absence of modulation of the differential signal at the PEM frequency of $50 \mathrm{kHz}$ clearly confirms the absence of circular dichroism. Figure 3(c) presents the results obtained by the same approach for samples rotated by $90^{\circ}$ around the $C_{4}$ axis. The fact that the differential signals $\Delta I_{B}^{(90)}(\phi)-\Delta I_{A}^{(90)}(\phi)$ and $\Delta I_{C}^{(90)}(\phi)-\Delta I_{A}^{(90)}(\phi)$ in Fig. 3(c) are phase-shifted by $\pi$ underscores the MLD nature of the differential signals.

The MLD-induced change in reflectivity is given by the differences between the maxima and minima of the traces in Figs. 3(b) and 3(c) multiplied by a factor of 2 [the signals shown in Figs. 3(b) and 3(c) are relative to the unpolarized reference sample A]. The reflectivity is found to be higher for the light polarized parallel to the staggered magnetization. The average value of the MLD-induced-reflectivity change from all data combined is $(0.52 \pm 0.14) \%$. We note, however, that it follows from Figs. 3(b) and 3(c) that the MLD extracted from $\Delta I_{B}(\phi)-$ $\Delta I_{A}(\phi)$ and $\Delta I_{B}^{(90)}(\phi)-\Delta I_{A}^{(90)}(\phi)[(0.63 \pm 0.03) \%]$ is larger than the MLD extracted from $\Delta I_{C}(\phi)-\Delta I_{A}(\phi)$ and $\Delta I_{C}^{(90)}(\phi)-\Delta I_{A}^{(90)}(\phi)[(0.41 \pm 0.07) \%]$. This suggests that the underlying microstructural strain favors the orientation of the Néel vector parallel to the $c$-axis tilt (parallel to the $[010]_{s}$ axis of the substrate), resulting in a partial polarization of the as-grown reference sample A along the $[010]_{s}$ axis. Indeed, a preliminary study using scanning electron microscopy with polarization analysis seems to support this observation [43].

Figure 3(d) presents results of the measurements of structural dichroism. Following Eq. (4), for differences $\Delta I_{A}(\phi)-\Delta I_{A}^{(90)}(\phi)$ and $\Delta I_{B}(\phi)-\Delta I_{C}^{(90)}(\phi)$ the signal due to the MLD should cancel out and the remaining variation of reflectivity is due to the structural/strain effects. The extracted $D_{\text {str }}=(0.26 \pm 0.08) \%$, with the reflectivity being larger for light polarized perpendicular to the direction of the $c$-axis tilt, i.e., parallel to the $[211]_{s}$ axis of the r-cut sapphire (see also Appendix B). Thus, the structural dichroism is about twice as small as the MLD in $\mathrm{Mn}_{2} \mathrm{Au}$ films on r-cut sapphire. It is noteworthy that the value of $D_{\text {str }}$ extracted from $\Delta I_{A}(\phi)-\Delta I_{A}^{(90)}(\phi)$ is again noticeably lower than the value extracted from $\Delta I_{B}(\phi)-$ $\Delta I_{C}^{(90)}(\phi)$. This is consistent with the above suggestion that the as-grown sample is partially polarized along the $[010]_{s}$ axis of the substrate, resulting in a lower value of the extracted structural dichroism when $\Delta I_{A}(\phi)-\Delta I_{A}^{(90)}(\phi)$ is monitored.

\section{CONCLUSIONS}

We demonstrate a pronounced magnetic linear dichroism in the NIR region in a collinear metallic antiferromagnet, $\mathrm{Mn}_{2} \mathrm{Au}$, at room temperature. The observed MLD of approximately $0.6 \%$ exceeds the value of the AMR in $\mathrm{Mn}_{2} \mathrm{Au}$ [22] recorded at liquid-helium temperatures by about a factor of 4 . While no study on the temperature dependence of the AMR in $\mathrm{Mn}_{2} \mathrm{Au}$ has been performed thus far, one may expect that the increased electron-phonon and electron-magnon scattering give rise to a suppression 
of AMR at higher temperatures, as typically observed. Thus, the ratio between the MLD and the AMR may be even higher at room temperature.

A recent broadband terahertz study of several ferromagnets demonstrated AMR signals up to $30 \mathrm{THz}$ [44], with the effect slowly decreasing with increasing frequency. The large difference between the observed MLD in the near-infrared region and the AMR in $\mathrm{Mn}_{2} \mathrm{Au}$ [22] implies the MLD in the near-infrared region is a result of the polarization-dependent interband absorption. Indeed, a recent angular-resolved photoemission spectroscopy study showed that the Néel-vector orientation leads to a pronounced valence-band asymmetry up to binding energies of several electronvolts [39]. Thus, further systematic studies of MLD as a function of photon energy may reveal spectral ranges with even higher MLD.

In view of the recent studies demonstrating a large magneto-optical contrast in ultrathin $\mathrm{NiO}$ [35-37] and $\mathrm{CoO}$ [38] films, it is tempting to estimate the polarization rotation angle, which corresponds to the observed MLDinduced reflectivity variation of approximately $0.6 \%$. Assuming the observed reflectivity change is dominated by the variation of the (interband) absorption coefficient, we estimate the polarization rotation angle for the light polarized $45^{\circ}$ from the Néel vector to be approximately 340 mdeg, about a factor of 2 larger than the reported value in $\mathrm{CoO}$ thin films [38] and more than a factor of 5 larger than the reported value in $\mathrm{NiO}$ thin films [35].

As a simple nonperturbative tabletop experiment, the approach presented can be extended to the imaging mode, enabling detection of the Néel-vector orientation with micrometer spatial resolution. Moreover, in view of potential applications of $\mathrm{Mn}_{2} \mathrm{Au}$ (e.g., as a spintronic memory device) use of sequences of femtosecond optical pulses could provide readout speeds matching the expected ultrafast switching times in antiferromagnetic memory devices.

\section{ACKNOWLEDGMENTS}

This work was funded by the Deutsche Forschungsgemeinschaft (DFG) under Grant No. TRR 173268565370 (Project A05, with additional contributions from A01). It received support from the Horizon 2020 framework program of the European Commission under Grant Agreement No. 863155 (S-NEBULA) and KAUST (OSR-2019CRG8-4048). V.G. and M.F. acknowledge financial support from the Graduate School of Excellence "Materials Science in Mainz" (DFG Grant No. GSC 266 49741853). We acknowledge the Paul Scherrer Institut, Villigen, Switzerland, for the beamtime allocation under proposal 20200977 at the SIM beamline of the Swiss Light Source. We thank the SIM beamline staff for technical support. We acknowledge valuable discussions with H.-J. Elmers, H. Gomonay, P. Grigorev, and L. Šmejkal.

\section{APPENDIX A: EXPERIMENTAL SETUP FOR OPTICAL DICHROISM}

Our experimental setup is sketched in Fig. 2(a). For the noncubic material, the experimental reflectivity anisotropy can be a result of a finite angle of incidence. For the same reason (i.e., the difference in reflectivities of $p$-polarized and $s$-polarized light), any dielectric mirrors need to be avoided in the beam path. Therefore, it is not possible to use normal incidence and to separate the reflected light with a semitransparent dielectric mirror. To minimize such effects, an angle of incidence of $\alpha / 2 \approx 0.34^{\circ}$ is used. Moreover, we use a minimal number of optical elements, with the only reflecting surface being the surface of the sample, as shown in Fig. 2(a).

We use a 852-nm laser diode and a PEM from Hinds Instruments, which operates at a frequency of $50 \mathrm{kHz}$. The PEM is set to $\lambda / 2$ retardation mode for $852 \mathrm{~nm}$, resulting in a modulation of light polarization between $s$ polarization (vertical) and $p$ polarization (horizontal) at $100 \mathrm{kHz}$, the second harmonic of the PEM; see Fig. 2(b).

The laser intensity is $5 \mathrm{~mW}$, while the diameter of the laser spot on the sample is $500 \mu \mathrm{m}$. The laser heating in thin films is governed by the absorbed light intensity and the thermal properties of the substrate (sapphire in our case). The temperature increase of the illuminated region can be estimated with a simple steady-state heat-diffusion model $[45,46]$. Using a reflectivity of 0.52 and the thermal conductivity of sapphire at room temperature of $35 \mathrm{~W} / \mathrm{mK}$ [47] we estimate, using Eq. (3) in Ref. [46], the steadystate heating to be less than $0.1 \mathrm{~K}$. Thus, laser heating can be ignored.

\section{APPENDIX B: POSSIBLE ORIGINS OF STRUCTURAL DICHROISM}

While our data clearly demonstrate the presence of a substantial MLD in $\mathrm{Mn}_{2} \mathrm{Au}$, there are two possible origins giving rise to the structural component of the measured reflectivity anisotropy; see Fig. 3(d). To address these, we first need to consider the specifics of the epitaxial film growth on r-cut sapphire using the $\mathrm{Ta}(001)$ buffer layer $[21,40]$.

As elaborated elsewhere [17,48], the $c$ axis of the $\mathrm{Mn}_{2} \mathrm{Au}$ films grown on $\mathrm{Al}_{2} \mathrm{O}_{3}(1 \overline{102})$ is tilted with respect to the surface normal by about $2-3^{\circ}$ (the tilt shows a small variation from sample to sample). The $c$ axis of the film tilts toward the $[010]_{s}$ axis of the substrate.

Given the tilted growth, the observed structural dichroism could be a result of the experimental geometry; the effect is illustrated in Fig. 4. Namely, because of the tilt of the film's $c$ axis with respect to the surface normal, the rotation of the sample by $90^{\circ}$ (or equivalently rotation of the light polarization) changes the projection of the $\vec{k}$ vector of light onto the $c$ axis. The difference between 


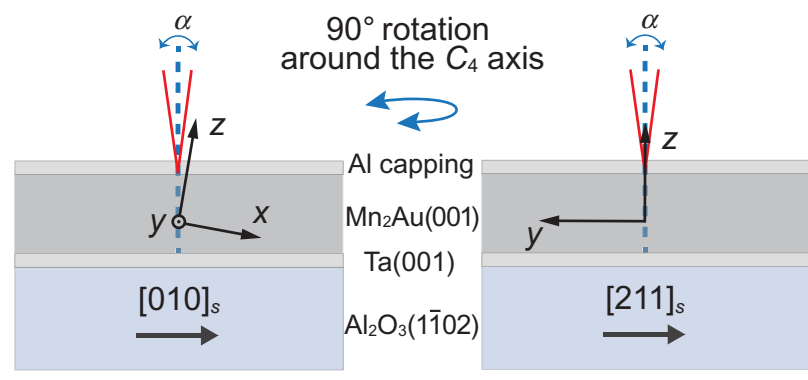

FIG. 4. The sample layout in dichroism measurements for the two different orientations of the sample (left and right) with respect to the laser-beam directions (red lines). Here $x$ and $y$ correspond to the [110] and [1 $\overline{10}$ ] directions of $\mathrm{Mn}_{2} \mathrm{Au}$, while $z$ is parallel to the $c$ axis, [001]. The tilt of the $c$ axis is highly exaggerated.

$\epsilon_{\perp}$ and $\epsilon_{\|}$can thus give rise to reflectivity modulation. Unfortunately, because of the lack of data on (anisotropic) optical properties of $\mathrm{Mn}_{2} \mathrm{Au}$, this contribution cannot be estimated.

On the other hand, the observed dichroism can also be a result of the underlying strain as a result of the anisotropic thermal expansion of sapphire [49,50]; such an effect on the low-symmetry cut of $\mathrm{Al}_{2} \mathrm{O}_{3}$ cannot be ignored. Indeed, investigations of sample morphology using atomic force microscopy suggest breaking of the fourfold symmetry [40]. If we consider the underlying strain to be responsible for the nonmagnetic components of dichroism, its amplitude can be roughly estimated from $D_{\text {str }}$. Assuming a linear relation between $D_{\text {str }}$ and the relative difference in the $[110] /[1 \overline{1} 0]$ lattice spacings, the latter should be on the order of $0.1 \%$. Using the XRD measurements, we are, however, unable to resolve such a weak asymmetry

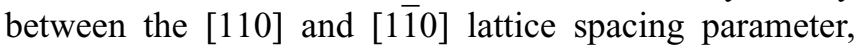
because of the rather large mosaicity of approximately $0.5^{\circ}$ $[21,40]$.

[1] I. Zutic, J. Fabian, and S. Das Sarma, Spintronics: Fundamentals and applications, Rev. Mod. Phys. 76, 323 (2004).

[2] A. H. MacDonald and M. Tsoi, Antiferromagnetic metal spintronics, Phil. Trans. R. Soc. A. 369, 3098 (2011).

[3] E. V. Gomonay and V. M. Loktev, Spintronics of antiferromagnetic systems (review article), Low Temp. Phys. 40, 17 (2014).

[4] T. Jungwirth, X. Marti, P. Wadley, and J. Wunderlich, Antiferromagnetic spintronics, Nat. Nanotech 11, 231 (2016).

[5] V. Baltz, A. Manchon, M. Tsoi, T. Moriyama, T. Ono, and Y. Tserkovnyak, Antiferromagnetic spintronics, Rev. Mod. Phys. 90, 015005 (2018).

[6] T. Kampfrath, A. Sell, G. Klatt, A. Pashkin, S. Mährlein, T. Dekorsy, M. Wolf, M. Fiebig, A. Leitenstorfer, and R. Huber, Coherent terahertz control of antiferromagnetic spin waves, Nat. Photonics 5, 31 (2011).
[7] A. H. M. Reid, T. Rasing, R. V. Pisarev, H. A. Dürr, and M. C. Hoffmann, Terahertz-driven magnetism dynamics in the orthoferrite $\mathrm{DyFeO}_{3}$, Appl. Phys. Lett. 106, 082403 (2015).

[8] K. Yamaguchi, M. Nakajima, and T. Suemoto, Coherent Control of Spin Precession Motion with Impulsive Magnetic Fields of Half-Cycle Terahertz Radiation, Phys. Rev. Lett. 105, 237201 (2010).

[9] T. H. Kim, S. Y. Hamh, J. W. Han, C. Kang, C.-S. Kee, S. Jung, J. Park, Y. Tokunaga, Y. Tokura, and J. S. Lee, Coherently controlled spin precession in canted antiferromagnetic $\mathrm{YFeO}_{3}$ using terahertz magnetic field, Appl. Phys. Express 7, 093007 (2014).

[10] M. Arana, F. Estrada, D. S. Maior, J. B. S. Mendes, L. E. Fernandez-Outon, W. A. A. Macedo, V. M. T. S. Barthem, D. Givord, A. Azevedo, and S. M. Rezende, Observation of magnons in $\mathrm{Mn}_{2} \mathrm{Au}$ films by inelastic Brillouin and Raman light scattering, Appl. Phys. Lett. 111, 192409 (2017).

[11] R. E. Camley, Z. Celinski, and R. L. Stamps, Magnetism of surfaces, interfaces, and nanoscale materials, Handbook Surf. Sci. 5, 2 (2015).

[12] J. Železnỳ, H. Gao, K. Vỳbornỳ, J. Zemen, J. Mašek, A. Manchon, J. Wunderlich, J. Sinova, and T. Jungwirth, Relativistic Néel-Order Fields Induced by Electrical Current in Antiferromagnets, Phys. Rev. Lett. 113, 157201 (2014).

[13] P. Wadley, B. Howells, J. Železnỳ, C. Andrews, V. Hills, R. P. Campion, V. Novák, K. Olejník, F. Maccherozzi, and S. Dhesi et al., Electrical switching of an antiferromagnet, Science 351, 587 (2016).

[14] M. J. Grzybowski, P. Wadley, K. W. Edmonds, R. Beardsley, V. Hills, R. P. Campion, B. L. Gallagher, J. S. Chauhan, V. Novak, T. Jungwirth, F. Maccherozzi, and S. S. Dhesi, Imaging Current-Induced Switching of Antiferromagnetic Domains in CuMnAs, Phys. Rev. Lett. 118, 057701 (2017).

[15] P. Wadley, S. Reimers, M. J. Grzybowski, C. Andrews, M. Wang, J. S. Chauhan, B. L. Gallagher, R. P. Campion, K. W. Edmonds, S. S. Dhesi, F. Maccherozzi, V. Novak, J. Wunderlich, and T. Jungwirth, Current polaritydependent manipulation of antiferromagnetic domains, Nat. Nanotechnol. 13, 362 (2018).

[16] S. Y. Bodnar, L. Šmejkal, I. Turek, T. Jungwirth, O. Gomonay, J. Sinova, A. A. Sapozhnik, H. J. Elmers, M. Kläui, and M. Jourdan, Writing and reading antiferromagnetic $\mathrm{Mn}_{2} \mathrm{Au}$ by Néel spin-orbit torques and large anisotropic magnetoresistance, Nat. Commun. 9, 348 (2018).

[17] S. Y. Bodnar, M. Filianina, S. P. Bommanaboyena, T. Forrest, F. Maccherozzi, A. Sapozhnik, Y. Skourski, M. Kläui, and M. Jourdan, Imaging of current induced Néel vector switching in antiferromagnetic $\mathrm{Mn}_{2} \mathrm{Au}$, Phys. Rev. B 99, 140409 (2019).

[18] M. Meinert, D. Graulich, and T. Matalla-Wagner, Electrical Switching of Antiferromagnetic $\mathrm{Mn}_{2} \mathrm{Au}$ and the Role of Thermal Activation, Phys. Rev. Appl. 9, 064040 (2018).

[19] K. Olejník, T. Seifert, Z. Kašpar, V. Novák, P. Wadley, R. P. Campion, M. Baumgartner, P. Gambardella, P. Němec, J. Wunderlich, J. Sinova, P. Kužel, M. Müller, T. Kampfrath, and T. Jungwirth, Terahertz electrical writing speed in an antiferromagnetic memory, Sci. Adv. 4, eaar3566 (2018). 
[20] V. Barthem, C. Colin, H. Mayaffre, M.-H. Julien, and D. Givord, Revealing the properties of $\mathrm{Mn}_{2} \mathrm{Au}$ for antiferromagnetic spintronics, Nat. Commun. 4, 1 (2013).

[21] M. Jourdan, H. Bräuning, A. Sapozhnik, H. Elmers, H. Zabel, and M. Kläui, Epitaxial $\mathrm{Mn}_{2} \mathrm{Au}$ thin films for antiferromagnetic spintronics, J. Phys. D: Appl. Phys. 48, 385001 (2015).

[22] S. Y. Bodnar, Y. Skourski, O. Gomonay, J. Sinova, M. Kläui, and M. Jourdan, Magnetoresistance Effects in the Metallic Antiferromagnet $\mathrm{Mn}_{2} \mathrm{Au}$, Phys. Rev. Appl. 14, 014004 (2020).

[23] P. Němec, M. Fiebig, T. Kampfrath, and A. V. Kimel, Antiferromagnetic opto-spintronics, Nat. Phys. 14, 229 (2018).

[24] S. A. Siddiqui, J. Sklenar, K. Kang, M. J. Gilbert, A. Schleife, N. Mason, and A. Hoffmann, Metallic antiferromagnets, J. Appl. Phys. 128, 040904 (2020).

[25] J. L. Erskine and E. A. Stern, Magneto-Optic Kerr Effect in Ni, Co, and Fe, Phys. Rev. Lett. 30, 1329 (1973).

[26] A. Kirilyuk, A. V. Kimel, and T. Rasing, Ultrafast optical manipulation of magnetic order, Rev. Mod. Phys. 82, 2731 (2010).

[27] T. Higo, H. Man, D. B. Gopman, L. Wu, T. Koretsune, O. M. van t Erve, Y. P. Kabanov, D. Rees, Y. Li, and M.-T. Suzuki et al., Large magneto-optical Kerr effect and imaging of magnetic octupole domains in an antiferromagnetic metal, Nat. Photonics 12, 73 (2018).

[28] A. S. Borovik-Romanov, A. A. Kreines, A. A. Pankov, and M. A. Talalayev, Magnetic birefringence of light in antiferromagnetic transition-metal fluorides, Sov. Phys.-JETP 64, 1762 (1973).

[29] B. B. Krichevtsov and R. V. Pisarev, Anisotropy of magnetic linear dichroism in cubic magnetic substances, Sov. Phys.-JETP 84, 865 (1983).

[30] J. Ferré and G. A. Gehring, Linear optical birefringence of magnetic crystals, Rep. Prog. Phys. 47, 513 (1984).

[31] V. Saidl, P. Němec, P. Wadley, V. Hills, R. Campion, V. Novák, K. Edmonds, F. Maccherozzi, S. Dhesi, and B. Gallagher et al., Optical determination of the Néel vector in a CuMnAs thin-film antiferromagnet, Nat. Photonics 11, 91 (2017).

[32] M. Surỳnek, V. Saidl, Z. Kašpar, V. Novák, R. Campion, P. Wadley, and P. Němec, Investigation of magnetic anisotropy and heat dissipation in thin films of compensated antiferromagnet CuMnAs by pump-probe experiment, J. Appl. Phys. 127, 233904 (2020).

[33] K. Yang, K. Kang, Z. Diao, A. Ramanathan, M. H. Karigerasi, D. P. Shoemaker, A. Schleife, and D. G. Cahill, Magneto-optic response of the metallic antiferromagnet $\mathrm{Fe}_{2} \mathrm{As}$ to ultrafast temperature excursions, Phys. Rev. Mater. 3, 124408 (2019).

[34] Z. Zheng, J. Y. Shi, Q. Li, T. Gu, H. Xia, L. Q. Shen, F. Jin, H. C. Yuan, Y. Z. Wu, L. Y. Chen, and H. B. Zhao, Magneto-optical probe of ultrafast spin dynamics in antiferromagnetic CoO thin films, Phys. Rev. B 98, 134409 (2018).

[35] J. Xu, C. Zhou, M. Jia, D. Shi, C. Liu, H. Chen, G. Chen, G. Zhang, Y. Liang, J. Li, W. Zhang, and Y. Wu, Imaging antiferromagnetic domains in nickel oxide thin films by optical birefringence effect, Phys. Rev. B 100, 134413 (2019).
[36] F. Schreiber, L. Baldrati, C. Schmitt, R. Ramos, E. Saitoh, R. Lebrun, and M. Kläui, Concurrent magnetooptical imaging and magneto-transport readout of electrical switching of insulating antiferromagnetic thin films, Appl. Phys. Lett. 117, 082401 (2020).

[37] H. Meer, F. Schreiber, C. Schmitt, R. Ramos, E. Saitoh, O. Gomonay, J. Sinova, L. Baldrati, and M. Kläui, Direct imaging of current-induced antiferromagnetic switching revealing a pure thermomagnetoelastic switching mechanism in NiO, Nano Lett. 21, 114 (2021).

[38] J. Xu, H. Chen, C. Zhou, D. Shi, G. Chen, and Y. $\mathrm{Wu}$, Optical imaging of antiferromagnetic domains in ultrathin $\mathrm{CoO}(001)$ films, New J. Phys. 22, 083033 (2020).

[39] H.-J. Elmers, S. Chernov, S. D'Souza, S. Bommanaboyena, S. Y. Bodnar, K. Medjanik, S. Babenkov, O. Fedchenko, D. Vasilyev, and S. Agustsson et al., Néel vector induced manipulation of valence states in the collinear antiferromagnet $\mathrm{Mn}_{2} \mathrm{Au}$, ACS Nano 14, 17554 (2020).

[40] S. Bommanaboyena, T. Bergfeldt, R. Heller, M. Kläui, and M. Jourdan, High quality epitaxial $\mathrm{Mn}_{2} \mathrm{Au}(001)$ thin films grown by molecular beam epitaxy, J. Appl. Phys. 127, 243901 (2020).

[41] A. Sapozhnik, M. Filianina, S. Y. Bodnar, A. Lamirand, M.-A. Mawass, Y. Skourski, H.-J. Elmers, H. Zabel, M. Kläui, and M. Jourdan, Direct imaging of antiferromagnetic domains in $\mathrm{Mn}_{2} \mathrm{Au}$ manipulated by high magnetic fields, Phys. Rev. B 97, 134429 (2018).

[42] A. A. Sapozhnik, R. Abrudan, Y. Skourski, M. Jourdan, H. Zabel, M. Kläui, and H.-J. Elmers, Manipulation of antiferromagnetic domain distribution in $\mathrm{Mn}_{2} \mathrm{Au}$ by ultrahigh magnetic fields and by strain, Phys. Status Solidi (RRL) Rapid Res. Lett. 11, 1600438 (2017).

[43] S. Bommanaboyena, D. Backes, L. Veiga, S. Dhesi, Y. Niu, B. Sarpi, T. Denneulin, A. Kovacs, T. Mashoff, and O. Gomonay et al., Readout of a antiferromagnetic spintronics systems by strong exchange coupling of $\mathrm{Mn}_{2} \mathrm{Au}$ and permalloy, arXiv:2106.02333 (2021).

[44] L. Nadvorník, M. Borchert, L. Brandt, R. Schlitz, K. A. de Mare, K. Vỳbornỳ, I. Mertig, G. Jakob, M. Kläui, and S. T. Goennenwein et al., Broadband Terahertz Probes of Anisotropic Magnetoresistance Disentangle Extrinsic and Intrinsic Contributions, Phys. Rev. X 11, 021030 (2021).

[45] H. S. Carslaw and J. C. Jaeger, Conduction of Heat in Solids (Clarendon press, Oxford, 1992).

[46] D. Mihailovic and J. Demsar, in Spectroscopy of Superconducting Materials (American Chemical Society, 1999), Chap. 16, p. 230.

[47] S. Burghartz and B. Schulz, Thermophysical properties of sapphire, $\mathrm{AlN}$ and $\mathrm{MgAl}_{2} \mathrm{O}_{4}$ down to $70 \mathrm{~K}$, J. Nucl. Mater. 212, 1065 (1994).

[48] S. Bodnar, Ph.D. thesis, Johannes Gutenberg-Universität Mainz (2020).

[49] W. M. Yim and R. J. Paff, Thermal expansion of AlN, sapphire, and silicon, J. Appl. Phys. 45, 1456 (1974).

[50] C. Roder, S. Einfeldt, S. Figge, T. Paskova, D. Hommel, P. P. Paskov, B. Monemar, U. Behn, B. A. Haskell, P. T. Fini, and S. Nakamura, Stress and wafer bending of a-plane GaN layers on r-plane sapphire substrates, J. Appl. Phys. 100, 103511 (2006). 